\title{
MICROWAVE RAPID HEATING SYSTEM USING CARBON HEATING TUBE
}

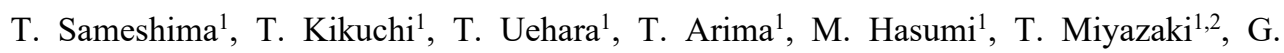
Kobayashi $^{2}$, and I. Serizawa ${ }^{3}$

${ }^{1}$ Tokyo University of Agriculture and Technology, Graduate School of Engineering, 2-2416, Naka-cho, Koganei, Tokyo, Japan

${ }^{2}$ Techno Research., Ltd., Koganei, Tokyo, Japan

${ }^{3}$ ORC MANUFACTUREING CO., LTD., Chino, Nagano, Japan

Email: tsamesim@cc.tuat.ac.jp

Keywords: Electromagnetic interference, Highly efficient heat treatment, PID temperature control, Silicon solar cell

A microwave heating system with a carbon heating tube (CHT) is reported. 2.45-GHz microwave at $200 \mathrm{~W}$ was introduced to a 300-dimameter metal cavity, in which the CHT made by $60-\mathrm{mm}$-long and $4-\mathrm{mm}$ diameter quartz tube filled with $2-\mu \mathrm{m}$ diameter carbon particles and $\mathrm{Ar}$ gas at $1400 \mathrm{~Pa}$ was set at the central position. The numerical simulation with a finite element moment method demonstrated the standing wave of the electric field caused by three-dimensional Fresnel interference effect. The lowest average electric field intensity of $5 \mathrm{kV} / \mathrm{m}$ in the cavity space was achieved in the case of the electrical conductivity of carbon ranging from 10 to $55 \mathrm{~S} / \mathrm{m}$. Heating a CHT with $55 \mathrm{~S} / \mathrm{m}$ was experimentally realized to $1200^{\circ} \mathrm{C}$ by microwave irradiation at $200 \mathrm{~W}$. This heating method was applied to activate $1.0 \times 10^{15}-\mathrm{cm}^{-2}$ boron and phosphorus implanted regions in n-type crystalline silicon substrate to fabricate pn junction and solar cells. The $\mathrm{CHT}$ heating at $1200^{\circ} \mathrm{C}$ realized a solar cell characteristic with a conversion efficiency of $14.8 \%$ under illumination of air mass 1.5 at $0.1 \mathrm{~W} / \mathrm{cm}^{2}$.

\section{Introduction}

Heat treatment has been widely applied for semiconductor device fabrication processing such as activation of dopant atoms to form the pn junction and crystallization to realize high quality crystalline state [1-6]. Many heating technologies have been developed such as laser annealing, plasma jet annealing, and rapid thermal annealing [7-10]. We have recently developed a lamp of carbon heating tube (CHT), in which 2- $\mu$ m-diameter-carbon powders were filled with inert gas in a quartz tube whose edges were then closed by thermal welding [11]. Conductive carbon is effectively heated via free carrier absorption of the electromagnetic energy with excellent thermal properties of low specific heat and high heat proof [12-14]. We have found that the low packing density of carbon powders about 0.09 allows effective absorption of microwave [12].

In this paper, we report characterization of the electric field distribution in the cavity in the steady state during microwave irradiation in the CHT heating system analyzed by a numerical simulator constructed with the three-dimensional finite element moment method to determine the effective condition of microwave absorption by the CHT. Then we demonstrate CHT heating with different temperatures controlled by a home-madeproportional-integral-differential feedback (PID) circuit which regulated the magnetron power source with a signal of the thermometer. We demonstrate application of the present heating system to activation of silicon surface region implanted with boron and phosphorus atoms in n-type silicon substrates. Decrease in the sheet resistivity and increase in the effective minority carrier lifetime $(\tau$ eff), and solar cell characteristics are reported. 


\section{Experimental}

\section{2-1. CHT heating system}

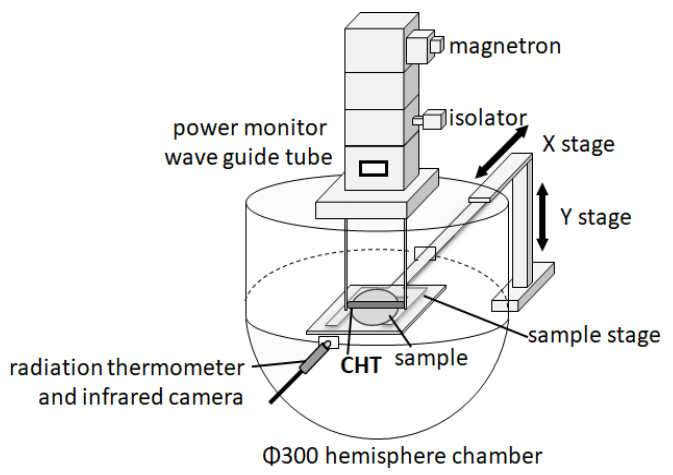

(a)

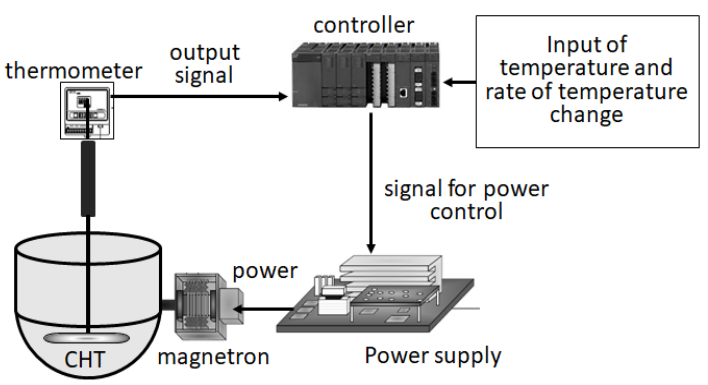

(b)

Fig. 1. Schematic images of (a) CHT heating system and (b) PID temperature control system.

Figure 1(a) shows a schematic image of the CHT heating system. For fabricating CHTs, quartz tubes with 60-mm long and 4-mm inter diameter and 6-mm outer diameter were prepared. $2-\mu \mathrm{m}$-diameter-carbon powders with a 0.1 -g weight were put in the quartz tubes, in which the packing density was controlled as 0.09 with an electrical conductivity of 55 $\mathrm{S} / \mathrm{m}$, which was determined by the previous work as the best value for heating [12]. Ar inert gas was filled in the quartz tube at $1400 \mathrm{~Pa}$. The edges of the quartz tubes were closed by thermal welding. Two quartz rods were jointed at the edges of the CHT by thermal welding to hold the CHT. A CHT was placed in the heating system which consists of a magnetron for generating 2.45-GHz microwave with an electrical power source, an isolator for blocking the reflectance of microwave backward the magnetron, a power monitor, an impedance matching box for reducing microwave reflectance, a wave-guide tube, and a microwave cavity made by Al metals. The cavity had a structure with a cylindrically upper part with a diameter of $300 \mathrm{~mm}$ and a spherically lower part to promote interferingly multiple reflection of the microwave in the cavity and to have the CHT effectively absorb the microwave. Those works resulted in a reflected power less than $4 \mathrm{~W}$ in the case of an input power of $500 \mathrm{~W}$. The CHT effectively absorbed the microwave power. In contrast, the power monitor gave the same value of the input and reflected power when the cavity had no CHT. The cavity completely reflected the microwave power. The cavity also had a window port for observing 
light emission from CHT heated by microwave. A thermometer detecting 900-nm wavelength radiation light CHINO IR-FAS was used to monitor the temperature of the CHT in real time. An infrared digital camera was also used to observe CHT heating behavior. A mechanically moving stage was also installed in the cavity, as shown in Fig. 1(a) to move a sample just below the CHT for demonstration of heating samples by the CHT. An electrical circuit was developed to control the CHT temperature, as shown by a schematic image in Fig. 1(b). The analog voltage signal of the thermometer is monitored in real time by a PID circuit controller [15] which was installed with a plan of temperature increasing and decreasing ratios, a target temperature and its duration in its memory in advance. The controller sent a signal to the power source of the magnetron to increase or decrease the microwave power for coinciding the $\mathrm{CHT}$ temperature with the initial temperature plan.

\section{2-2. Numerical calculation}

The electric field distribution in the cavity in the steady state during microwave irradiation was numerically analyzed by a simulator constructed with the three-dimensional finite element moment method combined with the Cholesky decomposition [16]. The grid system of the cavity and waveguide was formed with the equilateral-triangle elements with the sides ranging from 1 to $4 \mathrm{~mm}$ for the numerical calculation. They were assumed as perfect electric conductor which reflects completely the microwave. The CHT grid was formed with 1-mmside-equilateral-triangle elements. The electrical conductivity of carbon was widely changed from 0.1 to $10000 \mathrm{~S} / \mathrm{m}$ to find an effective condition of microwave absorption by the CHT. The calculation of electric field distribution in the cavity was also conducted in the case of no CHT.

\section{2-3. Solar cell fabrication}

4-inch-diameter 500 - $\mu$ m-thick $17-\Omega \mathrm{cm}$ n-type silicon substrates coated with 100 -nmthick thermally grown $\mathrm{SiO}_{2}$ layers were prepared. The ion implantation of boron atoms was conducted with a dose of $2.0 \times 10^{15} \mathrm{~cm}^{-2}$ at $25 \mathrm{keV}$ to the top silicon surface of the substrates. Phosphorus atoms with $2.0 \times 10^{15} \mathrm{~cm}^{-2}$ at $75 \mathrm{keV}$ was also implanted to the rear surface. The boron and phosphorus atoms with a dose of $1.0 \times 10^{15} \mathrm{~cm}^{-2}$ were effectively incorporated in the top and rear silicon surface regions. The samples were cut to 4 sectored pieces. They were placed in the heating system just below the CHT and moved at $0.12 \mathrm{~mm} / \mathrm{s}$. The CHT temperature was controlled by PID feedback method at 1000,1100 , and $1200^{\circ} \mathrm{C}$. After heating, the thermally grown $\mathrm{SiO}_{2}$ layer was removed using hydrofluoric acid. To estimate the sheet resistivity and effective minority carrier lifetime $\tau_{\text {eff, we measured the }} 9.35 \mathrm{GHz}$ microwave transmittance of the samples in dark field and 635-nm-light illumination [17]. Comb-type Al electrodes were formed on the top surface and the rear surface was entirely coated with $\mathrm{Al}$ electrodes by vacuum evaporation. 100-nm-thick $\mathrm{AlO}_{\mathrm{x}}$ layers was subsequently deposited on the top surfaces as anti-reflection layer. The samples were heated in $\mathrm{H}_{2} \mathrm{O}$ vaper at $0.8 \mathrm{MPa}$ at $230^{\circ} \mathrm{C}$ for $3 \mathrm{~h}$ to decrease defect states in the doped regions [18]. The electrical current density as a function of applied voltage $(\mathrm{J}-\mathrm{V})$ was measured under illumination of air mass (AM) 1.5 light at $0.1 \mathrm{~W} / \mathrm{cm}^{2}$.

\section{Results and discussion}

Figure 2 shows calculated cross-sectional electric field distributions in the cavity with different effective electrical conductivities of CHT of (a) 0 (no CHT), (b) 0.1, (c) 10, (d) 55, (e) 100 , and (f) $1000 \mathrm{~S} / \mathrm{cm}$ when the $200 \mathrm{~W}$ microwave was continuously introduced in the cavity. The calculation resulted in the standing wave of the electric field in every case because of the three-dimensional Fresnel interference effect in the cavity. The numerical 


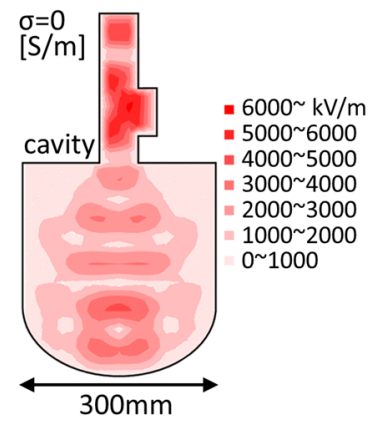

(a)

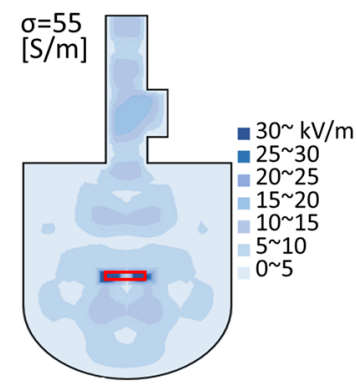

(d)

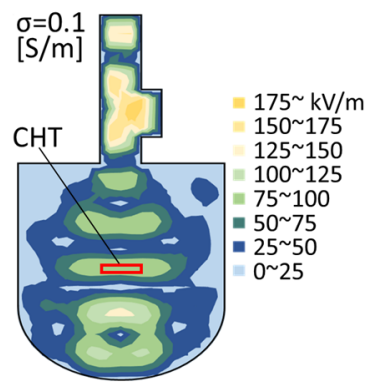

(b)

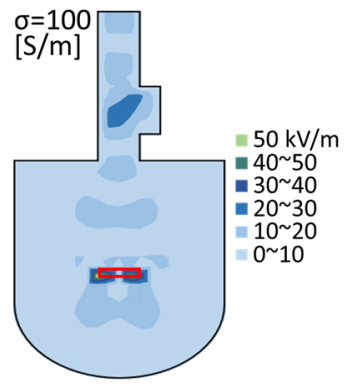

(e)

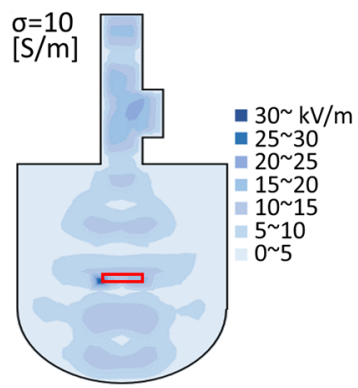

(c)

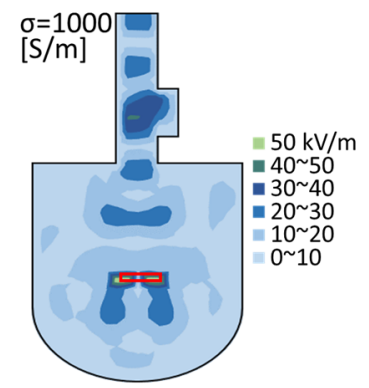

(f)

Fig. 2. Calculated cross-sectional electric field distributions with different effective electrical conductivities of CHT of (a) 0 (no CHT), (b) with 0.1 , (c) 10, (d) 55, (e) 100, and (f) $1000 \mathrm{~S} / \mathrm{cm}$ when $200 \mathrm{~W}$ microwave was continuously introduced.

calculation resulted in existence of a high electric field intensity ranging from 1000 to 6000 $\mathrm{kV} / \mathrm{m}$ in the no CHT case, as shown in Fig. 2(a). In contrast, the electric field intensity decreased to very low values in the case of the cavity with CHTs. In the most of region of the cavity, the electric field ranged from 25 to $125 \mathrm{kV} / \mathrm{m}$ for an electrical conductivity of 0.1 $\mathrm{S} / \mathrm{m}$, as shown in Fig. 2(b). This means that the microwave power was effectively absorbed by the CHT. The pattern of distribution of the electric field was similar to that of the no CHT case. Microwave was absorbed during three-dimensionally interfering in the cavity. The electric field intensity was very low when the electrical conductivity ranged from 10 to 100 $\mathrm{S} / \mathrm{m}$, as shown in Figs. 2(c) to (f). Especially the electric field intensity distributed from 5 to $25 \mathrm{kV} / \mathrm{m}$ in the case of 10 and 55-S/m-CHT, as shown in Figs. 2 (c) and (d). It was reduced to at least $0.1 \%$ of the initial intensity of the cavity space without CHT. This indicates that the microwave power in the cavity was almost completely absorbed by the CHT. Absorption of microwave power depends on the electrical conductivity. On the other hand, the electric field intensity increased when the electrical conductivity increased to $1000 \mathrm{~S} / \mathrm{m}$, as shown in Fig. 2(f). A CHT with a very high electrical conductivity (high packing density of carbon particles) is not suitable probably because of high reflectivity caused by a high extinction coefficient. 


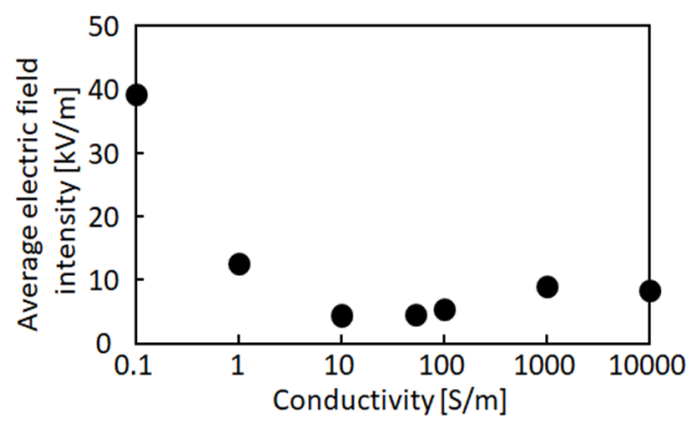

Fig. 3. Average electric field intensity in the cavity space as a function of the electrical conductivity of the CHT.

Figure 3 shows the average electric field intensity in the cavity space as a function of the electrical conductivity of the CHT. Although the average electric field intensity was very high of $1173 \mathrm{KV} / \mathrm{m}$ in the case of no CHT, it decreased to $40 \mathrm{kV} / \mathrm{m}$ by installing $0.1 \mathrm{~S} / \mathrm{m}$ $\mathrm{CHT}$, as shown in Fig. 3. The minimum average electric field intensity was $5 \mathrm{kV} / \mathrm{m}$ when the electrical conductivity ranged between 10 and $55 \mathrm{~S} / \mathrm{m}$. This means that the effective absorption of the microwave power by CHT reduced the electric field intensity in the cavity. The average electric field intensity gradually increased as the electrical conductivity further increased. The calculation supports experimental results of low microwave refection and effective heating of the CHT [11,12].

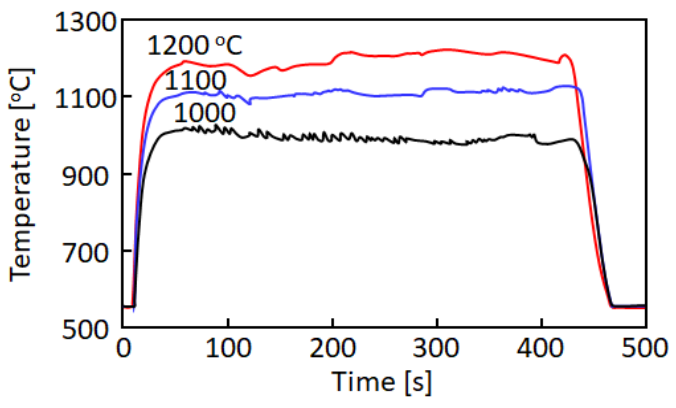

Fig. 4. Temperature changes with time of the CHT for three setting temperatures of 1000 , 1100 , and $1200^{\circ} \mathrm{C}$ during moving samples just below the $\mathrm{CHT}$ at $0.12 \mathrm{~mm} / \mathrm{s}$.

Figure 4 shows temperature changes with time of the $\mathrm{CHT}$ for three setting temperatures of 1000,1100 , and $1200^{\circ} \mathrm{C}$ under moving the silicon samples at $0.12 \mathrm{~mm} / \mathrm{s}$ just below the CHT. The PID control system well kept the setting temperatures by real-time changing the microwave power. Temperature fluctuation about $50 \mathrm{~K}$ was observed. This is probably because the condition of PID control with best time constant has not established yet. Further development of PID system is planned to precisely design temperature sequence for the CHT heating system. 


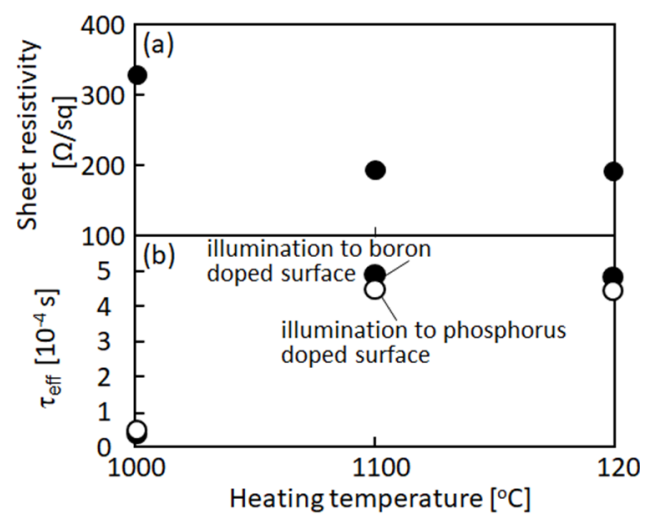

Fig. 5. (a) Sheet resistivity of the ionimplanted regions and (b) $\tau_{\text {eff }}$ in the case of 635-nm light illumination to the boron (solid circles) and phosphorus (open circles) doped surfaces.

Figure 5 shows (a) the sheet resistivity of the ion-implanted regions and (b) $\tau_{\text {eff }}$ in the case of 635-nm light illumination to the boron (solid circles) and phosphorus (open circles) implanted surfaces as functions of CHT heating temperature. The sheet resistivity of asimplanted sample was high of $626 \Omega$ /sq because of the low electron carrier density in the silicon bulk, it decreased to 215,148 , and $146 \Omega / \mathrm{sq}$, as the CHT heating temperature increased to 1000,1100 , and $1200^{\circ} \mathrm{C}$. Those experimental data resulted in that the implanted regions were activated with sheet resistivity of 327,194 , and $190 \Omega /$ sq, as shown in Fig. 5(a). The carrier generation were successfully achieved by CHT heating. $\tau_{\text {eff }}$ was also increased by CHT heating. Although they were low of $6.8 \times 10^{-6}$ and $9.1 \times 10^{-6} \mathrm{~s}$ in the cases of light illumination to the as-boron and as-phosphorus implanted surfaces, $\tau_{\text {eff }}$ increased to $3.3 \times 10^{-5}, 4.9 \times 10^{-4}$, and $4.8 \times 10^{-4} \mathrm{~s}$ in the cases of light illumination to the boron implanted surfaces as the CHT heating temperature increased to 1000,1100 , and $1200^{\circ} \mathrm{C}$. It also increased to $5.5 \times 10^{-5}, 4.5 \times 10^{-4}$, and $4.4 \times 10^{-4} \mathrm{~s}$ in the cases of light illumination to the phosphorus implanted surfaces as the CHT heating temperature increased to 1000,1100, and $1200^{\circ} \mathrm{C}$. These experimental lifetime results indicate that defect states caused by ion implantation were effectively reduced. Our numerical analysis resulted in that the recombination defect densities were $3.2 \times 10^{10}$ and $3.5 \times 10^{10} \mathrm{~cm}^{-2}$ for boron $\left(\mathrm{p}^{+}\right)$and phosphorus $\left(\mathrm{n}^{+}\right)$implanted surfaces in the case of $1200^{\circ} \mathrm{C} \mathrm{CHT}$ heating [17].

Figure 6 shows linear plotted J-V characteristics of solar cells fabricated with CHT heating at temperatures of 1000,1100 , and $1200^{\circ} \mathrm{C}$. Typical diode rectified characteristics were observed in dark field measurement. The AM 1.5 light illumination realized photovoltaic characteristics. The short circuit current density and open circuit voltage increased as the CHT heating temperature increased, as shown in Fig. 6. The conversion efficiency increased to $10.1,13.9$, and $14.8 \%$, as the CHT heating temperature increased. The $1200^{\circ} \mathrm{C}$ heating realized the best performance with the short circuit current density, open circuit voltage, fill factor, and conversion efficiency of $39 \mathrm{~mA} / \mathrm{cm}^{2}, 0.55 \mathrm{~V}, 0.69$, and $14.8 \%$, respectively. These results indicate that the temperature-controlled $\mathrm{CHT}$ heating has a capability of activating dopant-implanted regions, reducing the defect states, realizing high performance silicon solar cells. 


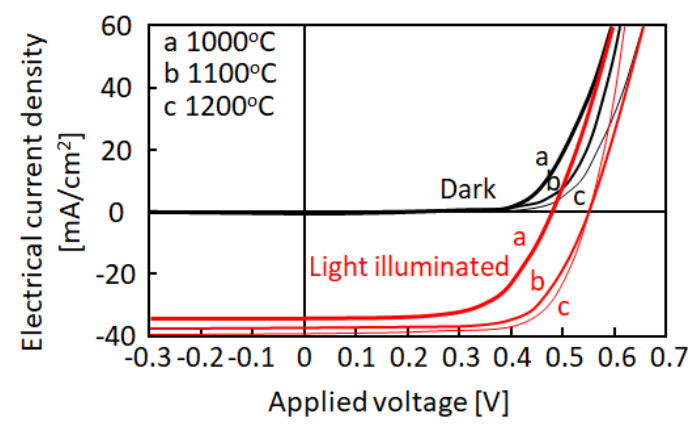

Fig. 6. Linear plotted J-V characteristics of solar cells.

\section{Conclusions}

We demonstrated CHT heating equipment which consisted of 2.45-GHz microwave irradiation wave guide tube into $300-\mathrm{mm}$-diametered semispherical metal cavity to accumulate the microwave, which was effectively absorbed by CHT made by a 4-mmdiameter and 60-mm-long quartz tube filled with conductive carbon particles at an average electrical conductivity of $55 \mathrm{~S} / \mathrm{m}$ in Ar gas at $1400 \mathrm{~Pa}$. The numerical simulation with a finite element moment method demonstrated the standing wave of the electric field caused by three-dimensional Fresnel interference effect. The lowest average electrical field intensity 5 $\mathrm{kV} / \mathrm{m}$ in the cavity space was achieved in the case of the electrical conductivity of carbon ranging from 10 to $55 \mathrm{~S} / \mathrm{m}$ when the $200 \mathrm{~W}$ microwave was continuously introduced. The PID feedback circuit regulated the microwave power by the signal of thermometer detecting the intensity of blackbody irradiation emitted from CHT heated by microwave to control the CHT heating temperature. The n-type silicon surface regions with $1.0 \times 10^{15} \mathrm{~cm}^{-2}$ boron and phosphorus ion implanted were heated at 1000,1100 , and $1200^{\circ} \mathrm{C}$ by $\mathrm{CHT}$ heating. The sheet resistivity of implanted regions decreased to $190 \Omega /$ sq by $1200^{\circ} \mathrm{C}$ heating and the density of defect states also decreased to $3.2 \times 10^{10}$ and $3.5 \times 10^{10} \mathrm{~cm}^{-2}$ for boron $\left(\mathrm{p}^{+}\right)$and phosphorus $\left(\mathrm{n}^{+}\right)$implanted surfaces. Via formation of $\mathrm{Al}$ metal electrodes and $\mathrm{AlO}_{\mathrm{x}^{-}}$ antireflection layer resulted in solar cell characteristics with the short circuit current density, open circuit voltage, fill factor, and conversion efficiency of $39 \mathrm{~mA} / \mathrm{cm}^{2}, 0.55 \mathrm{~V}, 0.69$, and $14.8 \%$, respectively, under illumination of air mass 1.5 at $0.1 \mathrm{~W} / \mathrm{cm}^{2}$.

\section{Acknowledgments}

This work was partially supported by Japan Science and Technology Agency ASTEP (No. AS3015022S).

\section{References}

1. S. M. Sze, Physics of Semiconductor Devices, 3rd ed., New York, WileyInterscience, 1985, 510-547.

2. Y. Taur, T, Ning, Fundamental of Modern VLSI Physics, 2nd ed., Cambridge, Cambridge University Press, 1998, 9-105.

3. S. Inoue, K. Sadao, T. Ozawa, Y. Kobashi, H. Kwai, T. Kitagawa, T. Shimoda, Tech. Dig. IEDM, 2000, 197.

4. K.Shibata, H. Takahashi, Workshop on Active Matrix Liquid Crystal Displays'01, 2001, 219-222. 
5. M. A. Green, Nat. Energy 1, 2016, Article number 15015.

6. M. Dahlinger, B. Bazer-Bachi, T. C. Röder, J. R. Köhler, R. Zapf-Gottwick, J. H. Werner, Energy Procedia, 2013, 38, 250-253.

7. T. Sameshima, S. Usui, M.Sekiya, IEEE Electron Device Lett., 1986, 7, 276-278.

8. T. Serikawa, S. Shirai, A. Okamoto, S. Suyama, Jpn. J. Appl. Phys., 1989, 28, L1871-L1873.

9. S. Higashi, H. Kaku H. Murakami, S. Miyazaki, H. Watakabe, N. Aando, T. Sameshima, 1st Thin Film Materials \& Devices Meeting, 2004, 25-28.

10. G. Mannino, Appl. Phys. Lett., 2001, 78, 889.

11. T. Sameshima, T. Miyazaki, G. Kobayashi, T. Arima, T. Kikuchi, T. Uehara, T. Sugawara, M. Hasumi, I. Serizawa, IEEE Access, 2019, 7, 23798-23805.

12. S. Kimura, K. Ota, M. Hasumi, A. Suzuki, M. Ushijima, T. Sameshima, Appl. Phys. $A, 2016, \mathbf{1 2 2}, 695$.

13. M. Yoshikawa, N. Nagai, M. Matsuki, H. Fukada, G. Katagiri, H. Ishida, A. Ishitani, I. Nagai, Phys. Rev. B, 1992, 46, 7169-7174.

14. Sameshima, N. Andoh, Jpn. J.Appl. Phys., 2005, 44, 7305-7308.

15. Aidan O'Dwyer, Handbook of PI and PID Controller Tuning Rules, 3rd ed., London, Imperial College Press, 2009, 4-17.

16. J. H. Richmond, IEEE Trans. Antennas \& Propagation, 1966, 14, 782-786.

17. T. Sameshima, H. Hayasaka, T. Haba, Jpn. J.Appl. Phys., 2009, 48, 021204-1-6.

18. T. Sameshima, M. Satoh, Jpn. J. Appl. Phys., 1997, 36, L687-L689. 